\title{
Spatial Search on Grids with Minimum Memory
}

\author{
Andris Ambainis ${ }^{1}$, Renato Portugal ${ }^{2}$, Nikolay Nahimov $^{1}$ \\ ${ }^{1}$ Faculty of Computing, University of Latvia, \\ Raina bulv. 19, Riga, LV-1586, Latvia \\ ${ }^{2}$ Laboratório Nacional de Computação Científica, \\ Av. Getúlio Vargas, 333, Petrópolis, 25651-075, Brazil
}

October 30,2018

\begin{abstract}
We study quantum algorithms for spatial search on finite dimensional grids. Patel et al. and Falk have proposed algorithms based on a quantum walk without a coin, with different operators applied at even and odd steps. Until now, such algorithms have been studied only using numerical simulations. In this paper, we present the first rigorous analysis for an algorithm of this type, showing that the optimal number of steps is $O(\sqrt{N \log N})$ and the success probability is $O(1 / \log N)$, where $N$ is the number of vertices. This matches the performance achieved by algorithms that use other forms of quantum walks.
\end{abstract}

\section{Introduction}

The quantum spatial search problem can be stated as follows. Suppose that one has a graph with $N$ vertices that represent the places that a quantum robot can be and the edges represent the directions that the robot can move among the vertices. Suppose also that one or a subset of vertices is marked. The goal is to find one marked vertex taking the least number of steps, assuming that the robot can move only to neighboring vertices, and each step takes one time unit.

Benioff [4] pointed out that a direct application of Grover's search algorithm [6] to the quantum spatial search problem on two-dimensional grids of size $\sqrt{N} \times \sqrt{N}$ does not provide a speedup compared to a search performed by a classical random walk, which takes $O(N \log N)$ steps. Aaronson and Ambainis [1] showed that most of quantum speedup can be recovered by using Grover's search together with a "divide-and-conquer" strategy that splits the grid into several subgrids and searches each of them. Using this method, the problem can be solved in $O\left(\sqrt{N} \log ^{2} N\right)$ steps.

The use of coined quantum walks [10] to the quantum spatial search problem was introduced by Shenvi et al. [11, which developed a quantum search algorithm for the hypercube taking $O(\sqrt{N})$ steps providing a quadratic speedup over classical method using random walk. Ambainis et al. (AKR) 3] used a similar method to build a quantum search algorithm on two-dimensional grids taking $O(\sqrt{N} \log N)$ steps using the method of amplitude amplification. By introducing an extra qubit into the system, Tulsi [12] was able to improve the time complexity of AKR's algorithm avoiding the use of amplitude amplification. Ambainis et al. (ABNOR) 2, also showed how to eliminate the method of amplitude amplification using the AKR's algorithm and performing a post-processing classical search.

Coinless (or staggered) quantum walks for hypercubic lattices were introduced by Patel et al. [8] by discretizing the Dirac equation used in the staggered lattice fermion formalism. The evolution operator is the product of two unitary operators, which are called even and 
odd, and can be obtained from shifted bases via a process of graph tessellation showed in Fig. 1] for the two dimensional case, which was pointed out by Falk [5]. Refs. 9] 7] also described the use of coinless quantum walks for searching on two-dimensional grids and concluded, using numerical implementations, that the search algorithm takes $O(\sqrt{N} \log N)$ steps without using Tulsi's method and $O(\sqrt{N \log N})$ with Tulsi's method. Using a similar algorithm, Falk concluded, also using numerical implementations, that the search algorithm takes $O(\sqrt{N})$ steps with constant success probability.

In this paper we analytically prove that a coinless quantum walk using the simplest tessellation (the same one used by Falk) takes $O(\sqrt{N \log N})$ steps to maximize the success probability, which depends on the grid size as $O(1 / \log N)$ when there is only one marked vertex. If we use the method of amplitude amplification, the total number of steps is $O(\sqrt{N} \log N)$ in order to achieve a constant success probability $\Theta(1)$.

The structure of this paper is the following: Sec. 2 describes the coinless quantum walk model on two-dimensional grids. Sec. 3 describes the general structure of the search algorithm, states two claims, and describes the algebraic manipulation necessary to prove the claims and to find the number of steps. Sec. 4describe the calculation of the number of steps that optimize the success probability. Sec. 5 describes the calculation of the norm of the main eigenvector of the evolution operator, which is used in the analysis of the algorithm. Secs. 6 and 7 prove the claims. In Sec. 8, we draw our conclusions and discuss possible extensions of this work.

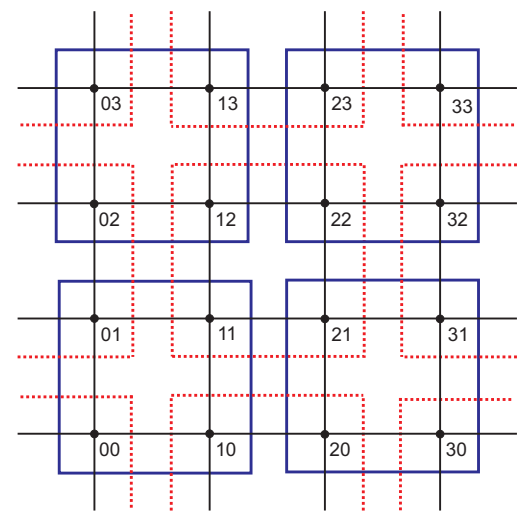

Figure 1: Grid tessellation using $2 \times 2$ cells. Blue squares (continuous line) represent the even tessellation and red squares (dotted line) represent the odd tessellation.

\section{Coinless Quantum Walks on Two-Dimensional Grids}

Consider a two-dimensional grid with $N$ vertices having a torus-like boundary conditions and the associated Hilbert space $\mathcal{H}^{N}$. We assume that $N$ is a perfect square and $\sqrt{N}$ is even. Define the set of orthonormal vectors

$$
\begin{aligned}
\left|u_{x y}^{\text {even }}\right\rangle & =\sum_{x^{\prime}, y^{\prime}=0}^{1} a_{x^{\prime}, y^{\prime}}\left|2 x+x^{\prime}, 2 y+y^{\prime}\right\rangle, \\
\left|u_{x y}^{\text {odd }}\right\rangle & =\sum_{x^{\prime}, y^{\prime}=0}^{1} b_{x^{\prime}, y^{\prime}}\left|2 x+x^{\prime}+1,2 y+y^{\prime}+1\right\rangle,
\end{aligned}
$$


which are based in Fig. 1. We address the case $a_{x^{\prime}, y^{\prime}}=b_{x^{\prime}, y^{\prime}}=1 / 2$. The projectors that project into the subspace spanned by $\left|u_{x y}^{\mathrm{e}}\right\rangle$ and $\left|u_{x y}^{\mathrm{o}}\right\rangle$ respectively are

$$
\begin{aligned}
& \Pi_{\mathrm{e}}=\sum_{x, y=0}^{\frac{1}{2} \sqrt{N}-1}\left|u_{x y}^{\mathrm{e}}\right\rangle\left\langle u_{x y}^{\mathrm{e}}\right|, \\
& \Pi_{\mathrm{o}}=\sum_{x, y=0}^{\frac{1}{2} \sqrt{N}-1}\left|u_{x y}^{\mathrm{o}}\right\rangle\left\langle u_{x y}^{\mathrm{o}}\right| .
\end{aligned}
$$

Define the reflection operators

$$
\begin{aligned}
& U_{\mathrm{e}}=2 \Pi_{\mathrm{e}}-I, \\
& U_{\mathrm{o}}=2 \Pi_{\mathrm{o}}-I .
\end{aligned}
$$

Define the reflection around the marked vertex

$$
U_{w}=2|w\rangle\langle w|-I .
$$

One step of the quantum walk is driven by the real unitary operator

$$
U=U_{\mathrm{o}} U_{w} U_{\mathrm{e}} U_{w}
$$

and the initial state is

$$
\left|\psi_{0}\right\rangle=\frac{1}{\sqrt{N}} \sum_{x, y=0}^{\sqrt{N}-1}|x, y\rangle .
$$

The algorithm consists in obtaining state $\left|\psi_{t_{f}}\right\rangle=U^{t_{f}}\left|\psi_{0}\right\rangle$, where $t_{f}$ is the number of steps, and performing a measurement in the computational basis. The result of the measurement is expected to be the marked vertex.

\section{Analysis of the Algorithm}

The eigenvalues of $U$ have the form $\exp ( \pm i \theta), 0 \leq \theta \leq \pi$. Among all eigenvalues different from 1, select the eigenvalue with the smallest positive argument. Let us denote this eigenvalue by $\exp (i \alpha)$ and the associated eigenvector by $|\psi\rangle$. Because $U$ is real, $\exp (-i \alpha)$ is also an eigenvalue and associated with eigenvector $|\psi\rangle^{*}$. Eigenvectors $|\psi\rangle$ and $|\psi\rangle^{*}$ are orthogonal and complex conjugates (the entries of $|\psi\rangle^{*}$ are the complex conjugate of the entries of $|\psi\rangle)$.

Define the vectors

$$
\begin{aligned}
& \left|\beta^{+}\right\rangle=\frac{1}{\sqrt{2} \||\psi\rangle \|}\left(|\psi\rangle+|\psi\rangle^{*}\right), \\
& \left|\beta^{-}\right\rangle=\frac{1}{\sqrt{2} \||\psi\rangle \|}\left(|\psi\rangle-|\psi\rangle^{*}\right),
\end{aligned}
$$

which are orthonormal. We claim that they define a plane in the Hilbert space $\mathcal{H}^{N}$, in which the state of algorithm approximately evolves. This statement is based in the following claims:

Claim 1 The overlap $\left|\left\langle\psi_{0} \mid \beta^{-}\right\rangle\right|$between the initial state and $\left|\beta^{-}\right\rangle$is $\Theta(1)$.

Claim 2 The success probability $p$ is $O\left(\frac{1}{\log N}\right)$.

Claim 1 says that if we replace the initial condition $\left|\psi_{0}\right\rangle$ by $\left|\beta^{-}\right\rangle$, the error will not increase when we increase $N$. The calculation of the evolution of the algorithm is simpler 
when we take $\left|\beta^{-}\right\rangle$as the intial condition, because $\left|\beta^{-}\right\rangle$is a linear combination of only two eigenvectors of the evolution operator, while $\left|\psi_{0}\right\rangle$ has overlap with all eigenvectors.

Suppose that $t_{f}$ is the number of steps fo the algorithm and take $\left|\beta^{-}\right\rangle$as the initial state, the final state will be

$$
\left|\psi_{f}\right\rangle=\frac{1}{\sqrt{2}}\left(\mathrm{e}^{i \alpha t_{f}}|\psi\rangle-\mathrm{e}^{-i \alpha t_{f}}|\psi\rangle^{*}\right) .
$$

If we take $t_{f}=\pi / 2 \alpha$, then $\left|\psi_{f}\right\rangle=i\left|\beta^{+}\right\rangle$, which is orthogonal to $\left|\beta^{-}\right\rangle$. The success probability is

$$
p=\left|\left\langle w \mid \beta^{+}\right\rangle\right|^{2} .
$$

The success probability decreases when we increase $N$, but Claim 2 states that the functional dependence is logarithmic. If one uses the method of amplitude amplification, the overhead to obtain a constant success probability is $O(\sqrt{\log N})$.

Let us define

$$
\begin{aligned}
& U_{1}=U_{\mathrm{e}} U_{w} U_{\mathrm{e}} U_{w}, \\
& U_{2}=U_{\mathrm{o}} U_{\mathrm{e}} .
\end{aligned}
$$

Using that $U_{\mathrm{e}}^{2}=I$, we have $U=U_{2} U_{1}$ with $U_{1}$ acting as follows: $U_{1}\left|\psi_{1}\right\rangle=\mathrm{e}^{2 \pi i / 3}\left|\psi_{1}\right\rangle$, $U_{1}\left|\psi_{2}\right\rangle=\mathrm{e}^{-2 \pi i / 3}\left|\psi_{2}\right\rangle$ and $U_{1}|\psi\rangle=|\psi\rangle$ if $|\psi\rangle \perp\left|\psi_{1}\right\rangle,|\psi\rangle \perp\left|\psi_{2}\right\rangle$. In our particular case, the vector $\left|\psi_{2}\right\rangle$ is the complex conjugate of $\left|\psi_{1}\right\rangle$ (i.e., all coefficients of $\left|\psi_{2}\right\rangle$ are complex conjugates of the corresponding coefficients of $\left|\psi_{1}\right\rangle$ ) and we will use this fact (see the appendix for details).

Let $\left|v_{j,+}\right\rangle$ and $\left|v_{j,-}\right\rangle$ (for $j=1,2, \ldots$ ) be the pairs of eigenvectors of $U_{2}$ with eigenvalues $\mathrm{e}^{i \theta_{j}}$ and $\mathrm{e}^{-i \theta_{j}}$ for $\theta_{j} \neq 0$ (and $\left|v_{j,+}\right\rangle$ is a complex conjugate of $\left|v_{j,-}\right\rangle$ ). We express

$$
\left|\psi_{1}\right\rangle=a\left|\psi_{1}^{\prime}\right\rangle+\sum_{j}\left(a_{j,+}\left|v_{j,+}\right\rangle+a_{j,-}\left|v_{j,-}\right\rangle\right)
$$

where $\left|\psi_{1}^{\prime}\right\rangle$ is an eigenvector of $U_{2}$ with eigenvalue 1. By taking complex conjugates of all coefficients of vectors on both sides of the equation, we get

$$
\left|\psi_{2}\right\rangle=a^{*}\left|\psi_{2}^{\prime}\right\rangle+\sum_{j}\left(a_{j,+}^{*}\left|v_{j,-}\right\rangle+a_{j,-}^{*}\left|v_{j,+}\right\rangle\right)
$$

where $\left|\psi_{2}^{\prime}\right\rangle$ is the complex conjugate of $\left|\psi_{1}^{\prime}\right\rangle$. The above equations are valid when $\sqrt{N} / 2$ is odd, because $U_{2}$ has no eigenvalue -1 in this case. Let $|\psi\rangle$ be the eigenvector of $U=U_{2} U_{1}$ with the eigenvalue $\mathrm{e}^{i \alpha}$ with the smallest positive $\alpha$. We multiply $|\psi\rangle$ by a constant so that $\left|\left\langle\psi_{1} \mid \psi\right\rangle\right|^{2}+\left|\left\langle\psi_{2} \mid \psi\right\rangle\right|^{2}=1$. Then, we can express

$$
|\psi\rangle=\cos \beta\left|\psi_{1}\right\rangle+x \sin \beta\left|\psi_{2}\right\rangle+\left|\psi^{\prime}\right\rangle
$$

where $\left|\psi^{\prime}\right\rangle \perp\left|\psi_{1}\right\rangle,\left|\psi^{\prime}\right\rangle \perp\left|\psi_{2}\right\rangle$ and $|x|=1$. To simplify the next expressions, we multiply $|\psi\rangle$ by a constant and $x$ by another constant so that

$$
|\psi\rangle=\mathrm{e}^{-i \pi / 3} \cos \beta\left|\psi_{1}\right\rangle+\mathrm{e}^{i \pi / 3} x \sin \beta\left|\psi_{2}\right\rangle+\left|\psi^{\prime}\right\rangle .
$$

Then,

$$
U_{1}|\psi\rangle=\mathrm{e}^{i \pi / 3} \cos \beta\left|\psi_{1}\right\rangle+\mathrm{e}^{-i \pi / 3} x \sin \beta\left|\psi_{2}\right\rangle+\left|\psi^{\prime}\right\rangle
$$

Let

$$
\begin{aligned}
|\varphi\rangle=U_{1}|\psi\rangle-|\psi\rangle= & \left(\mathrm{e}^{i \pi / 3}-\mathrm{e}^{-i \pi / 3}\right) \cos \beta\left|\psi_{1}\right\rangle+\left(\mathrm{e}^{-i \pi / 3}-\mathrm{e}^{i \pi / 3}\right) x \sin \beta\left|\psi_{2}\right\rangle \\
& =\sqrt{3} i \cos \beta\left|\psi_{1}\right\rangle-\sqrt{3} x i \sin \beta\left|\psi_{2}\right\rangle .
\end{aligned}
$$

By writing out $\left|\psi_{1}\right\rangle$ and $\left|\psi_{2}\right\rangle$ in terms of eigenvectors of $U_{2}$, we get

$$
|\varphi\rangle=\sqrt{3} i\left(\cos \beta\left|\psi_{1}\right\rangle-x \sin \beta\left|\psi_{2}\right\rangle\right)=\sqrt{3} i\left(a \cos \beta\left|\psi_{1}^{\prime}\right\rangle-a^{*} x \sin \beta\left|\psi_{2}^{\prime}\right\rangle\right.
$$




$$
\left.+\sum_{j}\left(\left(a_{j,+} \cos \beta-a_{j,-}^{*} x \sin \beta\right)\left|v_{j,+}\right\rangle+\left(a_{j,-} \cos \beta-a_{j,+}^{*} x \sin \beta\right)\left|v_{j,-}\right\rangle\right)\right) .
$$

Let $\left|\varphi^{\prime}\right\rangle=U_{1}|\psi\rangle+|\psi\rangle$. Then, we have

$$
|\psi\rangle=\frac{1}{2}\left|\varphi^{\prime}\right\rangle-\frac{1}{2}|\varphi\rangle, \quad U_{1}|\psi\rangle=\frac{1}{2}\left|\varphi^{\prime}\right\rangle+\frac{1}{2}|\varphi\rangle .
$$

To get $U_{2} U_{1}|\psi\rangle=\mathrm{e}^{i \alpha}|\psi\rangle$, we must have

$$
\begin{gathered}
\left|\varphi^{\prime}\right\rangle=\sqrt{3} \cot \frac{\alpha}{2}\left(a \cos \beta\left|\psi_{1}^{\prime}\right\rangle-a^{*} x \sin \beta\left|\psi_{2}^{\prime}\right\rangle\right) \\
+\sqrt{3} \sum_{j} \cot \frac{\alpha-\theta_{j}}{2}\left(a_{j,+} \cos \beta-a_{j,-}^{*} x \sin \beta\right)\left|v_{j,+}\right\rangle \\
+\sqrt{3} \sum_{j} \cot \frac{\alpha+\theta_{j}}{2}\left(a_{j,-} \cos \beta-a_{j,+}^{*} x \sin \beta\right)\left|v_{j,-}\right\rangle .
\end{gathered}
$$

Because of equation (15), we have

$$
\left\langle\psi_{1} \mid \psi\right\rangle=\mathrm{e}^{-i \pi / 3} \cos \beta=\left(\frac{1}{2}-\frac{\sqrt{3}}{2} i\right) \cos \beta .
$$

By combining this with the first part of (18) and (16), we get that $\left\langle\psi_{1} \mid \varphi^{\prime}\right\rangle=\cos \beta$. Similarly, $\left\langle\psi_{2} \mid \varphi^{\prime}\right\rangle=x \sin \beta$. By writing out $\left|\psi_{1}\right\rangle,\left|\psi_{2}\right\rangle$ and $\left|\varphi^{\prime}\right\rangle$ in terms of eigenvectors of $U_{2}$, we get

$$
\begin{gathered}
\left\langle\psi_{1} \mid \varphi^{\prime}\right\rangle=\sqrt{3} \cot \frac{\alpha}{2}\left(|a|^{2} \cos \beta-\left(a^{*}\right)^{2} x \sin \beta\left\langle\psi_{1}^{\prime} \mid \psi_{2}^{\prime}\right\rangle\right) \\
+\sqrt{3} \sum_{j} \cot \frac{\alpha-\theta_{j}}{2}\left(\left|a_{j,+}\right|^{2} \cos \beta-a_{j,+}^{*} a_{j,-}^{*} x \sin \beta\right) \\
+\sqrt{3} \sum_{j} \cot \frac{\alpha+\theta_{j}}{2}\left(\left|a_{j,-}\right|^{2} \cos \beta-a_{j,-}^{*} a_{j,+}^{*} x \sin \beta\right)=\cos \beta
\end{gathered}
$$

and

$$
\begin{gathered}
\left\langle\psi_{2} \mid \varphi^{\prime}\right\rangle=\sqrt{3} \cot \frac{\alpha}{2}\left(-|a|^{2} x \sin \beta+a^{2} \cos \beta\left\langle\psi_{2}^{\prime} \mid \psi_{1}^{\prime}\right\rangle\right) \\
+\sqrt{3} \sum_{j} \cot \frac{\alpha-\theta_{j}}{2}\left(a_{j,-} a_{j,+} \cos \beta-\left|a_{j,-}\right|^{2} x \sin \beta\right) \\
+\sqrt{3} \sum_{j} \cot \frac{\alpha+\theta_{j}}{2}\left(a_{j,+} a_{j,-} \cos \beta-\left|a_{j,+}\right|^{2} x \sin \beta\right)=x \sin \beta .
\end{gathered}
$$

\section{Number of Steps}

As described in Sec. 3, the number of steps of the algorithm is $\pi / 2 \alpha$. The determination of the asymptotic (large $N$ ) value of parameter $\alpha$ is the main part to describe the algorithm efficiency. We address this issue in this section.

We take the complex conjugate of both sides of (20) and rewrite the resulting equation as

$$
\sqrt{3} A_{11} \cos \beta+\sqrt{3} A_{12} x^{*} \sin \beta=\cos \beta
$$

where

$$
A_{11}=|a|^{2} \cot \frac{\alpha}{2}+\sum_{j}\left|a_{j,+}\right|^{2} \cot \frac{\alpha-\theta_{j}}{2}+\sum_{j}\left|a_{j,-}\right|^{2} \cot \frac{\alpha+\theta_{j}}{2}
$$




$$
A_{12}=-a^{2}\left\langle\psi_{2}^{\prime} \mid \psi_{1}^{\prime}\right\rangle \cot \frac{\alpha}{2}-\sum_{j} a_{j,+} a_{j,-}\left(\cot \frac{\alpha-\theta_{j}}{2}+\cot \frac{\alpha+\theta_{j}}{2}\right) .
$$

We can show that, for any $\theta \neq 0, \sum_{j: \theta_{j}=\theta}\left|a_{j,+}\right|^{2}=\sum_{j: \theta_{j}=\theta}\left|a_{j,-}\right|^{2}$. Therefore, we can simplify $A_{11}$ to

$$
A_{11}=|a|^{2} \cot \frac{\alpha}{2}+\sum_{j} \frac{\left|a_{j,+}\right|^{2}+\left|a_{j,-}\right|^{2}}{2}\left(\cot \frac{\alpha-\theta_{j}}{2}+\cot \frac{\alpha+\theta_{j}}{2}\right) .
$$

We can also rewrite (21) using that $|x|=1$ as

$$
-\sqrt{3} A_{11} \sin \beta-\sqrt{3} A_{12} x^{*} \cos \beta=\sin \beta .
$$

For $\alpha$ close to 0 , we can use the approximations $\cot x \approx \frac{1}{x}$ for $\cot \frac{\alpha}{2}$ and

$$
\cot \frac{\alpha-\theta_{j}}{2}+\cot \frac{\alpha+\theta_{j}}{2} \approx-\frac{\alpha}{\sin ^{2}\left(\theta_{j} / 2\right)} .
$$

Notice that using Eq. (34) from the appendix we conclude that the minimum positive value of $\theta_{j}$ is $4 \pi / \sqrt{N}$. We are going to show that $\alpha \ll \theta_{j}$ for large $N$. Under those approximations, we obtain

$$
\begin{gathered}
A_{11} \approx \frac{2|a|^{2}}{\alpha}-\alpha B, \\
A_{12} \approx-\frac{2 a^{2}\left\langle\psi_{2}^{\prime} \mid \psi_{1}^{\prime}\right\rangle}{\alpha}+\alpha C .
\end{gathered}
$$

where

$$
\begin{gathered}
B=\sum_{j} \frac{1}{2 \sin ^{2}\left(\theta_{j} / 2\right)}\left(\left|a_{j,+}\right|^{2}+\left|a_{j,-}\right|^{2}\right), \\
C=\sum_{j} \frac{1}{\sin ^{2}\left(\theta_{j} / 2\right)} a_{j,-} a_{j,+} .
\end{gathered}
$$

By eliminating $A_{12} x^{*}$ from Eqs. (22) and (23), we obtain

$$
\cos \beta=\frac{1}{\sqrt{2}}\left(1+\frac{1}{\sqrt{3} A_{11}}\right)^{\frac{1}{2}} .
$$

By multiplying $(-\sin \beta)$ to Eq. (22) and adding to Eq. (23) times $\cos \beta$, we obtain

$$
A_{11} \sin 2 \beta+A_{12} x^{*}=0 .
$$

Using the last expressions we have obtained for $A_{11}$ and $A_{12}$, we get

$$
\alpha^{2}=\frac{2|a|^{2} \sin 2 \beta-2 a^{2}\left\langle\psi_{2}^{\prime} \mid \psi_{1}^{\prime}\right\rangle x^{*}}{B \sin 2 \beta-C x^{*}} .
$$

The leading term (zeroth order in $N$ ) in the numerator of $\alpha^{2}$ is zero if $x=|a|^{2} / a^{* 2}\left\langle\psi_{1}^{\prime} \mid \psi_{2}^{\prime}\right\rangle$. We use this fact to calculate the value of $x$. Using the eigenvectors and eigenvalues of $U_{2}$ given in the appendix, we obtain

$$
|a|^{2}=\frac{1}{3}+\frac{8}{3 N}+O\left(\frac{1}{N^{2}}\right)
$$

and

$$
a^{2}\left\langle\psi_{2}^{\prime} \mid \psi_{1}^{\prime}\right\rangle=\mathrm{e}^{\frac{2 \pi i}{3}}\left(\frac{1}{3}-\frac{4}{3 N}\right)+O\left(\frac{1}{N^{2}}\right)
$$


Using that $\alpha^{2} B \ll|a|^{2}$ for large $N$, we can consider $A_{11} \approx 2|a|^{2} / \alpha$ and

$$
\cos \beta \approx \frac{1}{\sqrt{2}}\left(1+\frac{\sqrt{3} \alpha}{4}\right) .
$$

Similarly, using that $\sin 2 \beta \approx 1$, the first order approximation for $\alpha$ when $N$ is large is

$$
\alpha^{2} \approx \frac{8}{N\left(B-C x^{*}\right)} .
$$

Using the eigenvectors and eigenvalues of $U_{2}$, we obtain

$$
B-C x^{*}=\frac{2}{N} \sum_{\substack{k, l=0 \\ k, l) \neq(0,0)}}^{\frac{\sqrt{N}}{2}-1} \frac{1}{1-\cos ^{2} \tilde{k} \cos ^{2} \tilde{l}}
$$

where $\tilde{k}=2 \pi k / \sqrt{N}$ and $\tilde{l}=2 \pi l / \sqrt{N}$. Converting the double sum to a double integral and using residues (the expression inside the double sum taken as a function of $\tilde{k}$ and $\tilde{l}$ in the domain $(0, \pi)$ has four positive poles), we obtain $B-C x^{*}=O(\log N)$. Using this result, we conclude that

$$
\alpha=O\left(\frac{1}{\sqrt{N \log N}}\right) .
$$

\section{The Norm of $|\psi\rangle$}

Using Eqs. (17) and (19), we obtain

$$
\begin{gathered}
|\psi\rangle=\frac{\sqrt{3}}{2}\left(\cot \frac{\alpha}{2}-i\right)\left(a \cos \beta\left|\psi_{1}^{\prime}\right\rangle-a^{*} x \sin \beta\left|\psi_{2}^{\prime}\right\rangle\right) \\
+\frac{\sqrt{3}}{2} \sum_{j}\left(\cot \frac{\alpha-\theta_{j}}{2}-i\right)\left(a_{j,+} \cos \beta-a_{j,-}^{*} x \sin \beta\right)\left|v_{j,+}\right\rangle \\
+\frac{\sqrt{3}}{2} \sum_{j}\left(\cot \frac{\alpha+\theta_{j}}{2}-i\right)\left(a_{j,-} \cos \beta-a_{j,+}^{*} x \sin \beta\right)\left|v_{j,-}\right\rangle .
\end{gathered}
$$

By employing the approximation for small $\alpha$

$$
\cot ^{2} \frac{\alpha \pm \theta_{j}}{2}+1 \approx \frac{1}{\sin ^{2} \frac{\theta_{j}}{2}} \mp \frac{2 \sin \theta_{j} \alpha}{\left(1-\cos \theta_{j}\right)^{2}} .
$$

we obtain

$$
\begin{gathered}
\langle\psi \mid \psi\rangle \approx \frac{3}{4}\left(|a|^{2}-a^{2}\left\langle\psi_{2}^{\prime} \mid \psi_{1}^{\prime}\right\rangle x^{*} \sin 2 \beta\right)\left(\cot ^{2} \frac{\alpha}{2}+1\right) \\
+\frac{3}{4} \sum_{j} \frac{1}{\sin \frac{\theta_{j}}{2}}\left(\left|a_{j,+}\right|^{2}+\left|a_{j,-}\right|^{2}-2 \Re\left(a_{j,-} a_{j,+} x^{*}\right) \sin 2 \beta\right) .
\end{gathered}
$$

Using that $\sin 2 \beta \approx 1$, Eqs. (24) and (25), we obtain

$$
\langle\psi \mid \psi\rangle \approx \frac{12}{N \alpha^{2}}+\frac{3}{2}\left(B-C x^{*}\right) .
$$

Using Eq. (27), we get

$$
\langle\psi \mid \psi\rangle \approx \frac{24}{N \alpha^{2}} .
$$

Therefore, $\||\psi\rangle \|=O(\sqrt{\log N})$. 


\section{Proof of Claim 1}

Let $\left|\psi_{0}\right\rangle$ be the normalized uniform vector (initial condition of the algorithm). We know that $\left\langle\psi_{0} \mid v_{j, \pm}\right\rangle=0$, then for small $\alpha$

$$
\left\langle\psi_{0} \mid \psi\right\rangle \approx \frac{\sqrt{3}}{\sqrt{2} \alpha}\left(a\left\langle\psi_{0} \mid \psi_{1}^{\prime}\right\rangle-a^{*}\left\langle\psi_{0} \mid \psi_{2}^{\prime}\right\rangle x\right) .
$$

Using Eqs. (13) and (14) we conclude that $a\left\langle\psi_{0} \mid \psi_{1}^{\prime}\right\rangle=\left\langle\psi_{0} \mid \psi_{1}\right\rangle$ and $a^{*}\left\langle\psi_{0} \mid \psi_{2}^{\prime}\right\rangle=\left\langle\psi_{0} \mid \psi_{2}\right\rangle$. By replacing those values into the last equation and using that $x=\mathrm{e}^{2 \pi i / 3}$, we obtain

$$
\left\langle\psi_{0} \mid \psi\right\rangle \approx \frac{\sqrt{3}}{\sqrt{N} \alpha}(\sqrt{3}-i) .
$$

Using Eq. (29), we conclude that the overlap between the initial condition and the normalized vector $|\psi\rangle-|\psi\rangle^{*}$ is

$$
\frac{\left|\left\langle\psi_{0} \mid \psi\right\rangle-\left\langle\psi_{0} \mid \psi\right\rangle^{*}\right|}{\sqrt{2} \||\psi\rangle \|}=\Theta(1)
$$

The asymptotic overlap in this case is $1 / 2$. This overlap can be improved by changing the global phase of $|\psi\rangle$. In fact, if we take $\mathrm{e}^{-\pi i / 3}|\psi\rangle$, the asymptotic overlap is 1 .

\section{Proof of Claim 2}

Let $|00\rangle$ be the marked vertex. From Eq. (13), we obtain

$$
a\left\langle 00 \mid \psi_{1}^{\prime}\right\rangle=\left\langle 00 \mid \psi_{1}\right\rangle-\sum_{j}\left(a_{j,+}\left\langle 00 \mid v_{j,+}\right\rangle-a_{j,-}\left\langle 00 \mid v_{j,-}\right\rangle\right)
$$

A similar equation can be obtained for $a\left\langle 00 \mid \psi_{2}^{\prime}\right\rangle$ using Eq. (14). By employing those results, the overlap between the marked vertex and vector $|\psi\rangle$ can be written as

$$
\begin{gathered}
\langle 00 \mid \psi\rangle=\frac{\sqrt{3}}{2}\left(\left(\cot \frac{\alpha}{2}-i\right)\left(\cos \beta\left\langle 00 \mid \psi_{1}\right\rangle-x \sin \beta\left\langle 00 \mid \psi_{2}\right\rangle\right)\right. \\
+\sum_{j}\left(\cot \frac{\alpha-\theta_{j}}{2}-\cot \frac{\alpha}{2}\right)\left(a_{j,+} \cos \beta-a_{j,-}^{*} x \sin \beta\right)\left\langle 00 \mid v_{j,+}\right\rangle \\
\left.+\sum_{j}\left(\cot \frac{\alpha+\theta_{j}}{2}-\cot \frac{\alpha}{2}\right)\left(a_{j,-} \cos \beta-a_{j,+}^{*} x \sin \beta\right)\left\langle 00 \mid v_{j,-}\right\rangle\right) .
\end{gathered}
$$

By Taylor expanding $\cot \frac{\alpha \pm \theta_{j}}{2}$ around $\alpha=0$, using $\cot \frac{\alpha}{2} \approx \frac{2}{\alpha}$, Eq. (26), and discarding terms proportional to $\alpha$, we obtain

$$
\langle 00 \mid \psi\rangle \approx \frac{5 \sqrt{3} x^{*}}{8}+\frac{\sqrt{3}}{\sqrt{2} \alpha}\left(\frac{i x^{*}}{\sqrt{2}}-E^{-}\right)-\frac{3}{4 \sqrt{2}} E^{+}-\frac{\sqrt{3}}{2 \sqrt{2}} F
$$

where

$$
E^{ \pm}=\sum_{j}\left(\left(a_{j,+} \pm a_{j,-}^{*} x\right)\left\langle 00 \mid v_{j,+}\right\rangle+\left(a_{j,-} \pm a_{j,+}^{*} x\right)\left\langle 00 \mid v_{j,-}\right\rangle\right)
$$

and

$$
F=\sum_{j} \cot \frac{\theta_{j}}{2}\left(\left(a_{j,+}-a_{j,-}^{*} x\right)\left\langle 00 \mid v_{j,+}\right\rangle-\left(a_{j,-}-a_{j,+}^{*} x\right)\left\langle 00 \mid v_{j,-}\right\rangle\right) .
$$


By employing the expressions for $a_{j, \pm}$ and $\left|v_{j, \pm}\right\rangle$ given in the appendix, it is straightforward to show that

$$
\begin{gathered}
E^{-}=\frac{\sqrt{2}(\sqrt{3}-i)}{N} \sum_{\substack{k, l=0 \\
k, l) \neq(0,0)}}^{\frac{\sqrt{N}}{2}-1}\left(1-\frac{\epsilon \sin (\tilde{k}+\tilde{l})}{\sqrt{1-\cos ^{2} \tilde{k} \cos ^{2} \tilde{l}}}\right), \\
E^{+}=-\frac{i}{\sqrt{3}} E^{-}-\frac{1+i \sqrt{3}}{N \sqrt{6}} \sum_{\substack{k, l=0 \\
(k, l) \neq(0,0)}}^{\frac{\sqrt{N}}{2}-1} \frac{\sin 2 \tilde{k} \sin 2 \tilde{l}}{1-\cos ^{2} \tilde{k} \cos ^{2} \tilde{l}}, \\
F=\frac{\sqrt{2}(1+i \sqrt{3})}{N} \sum_{\substack{k, l=0 \\
(k, l) \neq(0,0)}}^{\frac{\sqrt{N}}{2}-1} \frac{\epsilon \sin \tilde{k} \sin \tilde{l}}{1-\cos ^{2} \tilde{k} \cos ^{2} \tilde{l}},
\end{gathered}
$$

where $\epsilon$ is the $\operatorname{sign}$ of $\cos \tilde{k} \cos \tilde{l}$. Calculating the double sums, we obtain

$$
\begin{gathered}
E^{-}=\frac{\sqrt{3}-i}{2 \sqrt{2}}\left(1-\frac{4}{N}\right), \\
E^{+}=-\frac{1+i \sqrt{3}}{2 \sqrt{6}}\left(1-\frac{4}{N}\right),
\end{gathered}
$$

and $F=0$. By replacing those results into Eq. (30), we obtain

$$
\langle 00 \mid \psi\rangle \approx-\frac{\sqrt{3}(1+i \sqrt{3})}{4}\left(1+\frac{1}{N}\right)+\frac{\sqrt{3}(\sqrt{3}-i)}{N \alpha} .
$$

For large $N$, the real part of the overlap $\langle 00 \mid \psi\rangle$ tends to $-\sqrt{3} / 4$. By using the fact that $\||\psi\rangle \|=O(\sqrt{\log N})$, we conclude that the modulus of the overlap between the marked vertex and the normalized vector $|\psi\rangle+|\psi\rangle^{*}$ is

$$
\frac{\left|\langle 00 \mid \psi\rangle+\langle 00 \mid \psi\rangle^{*}\right|}{\sqrt{2} \||\psi\rangle \|}=O\left(\frac{1}{\sqrt{\log N}}\right) .
$$

\section{Conclusions and Discussions}

We have analyzed the spatial search problem on two-dimensional grids using the coinless (or staggered) quantum walk model introduced by Patel et al. $[8$. We obtain the asymptotic (large $N$ ) number of step of the algorithm and the asymptotic success probability. We have used the simplest grid tessellation. As described in Fig. 1, we divide the the grid in $2 \times 2$ cells having the even-even points in the lowest left corner of the cells, which provides the even tessellation. The odd tessellation is obtained by displacing the even tessellation along the diagonal, so that odd-odd points are in the lowest left corner. Each cell in the even tessellation is associated with a normalized uniform vector in Hilbert space $\mathcal{H}^{N}$, which span a Hilbert subspace of dimension $N / 4$. Non-uniform basis vectors can be used paying a high price in terms of algebraic manipulations. The unitary operator $U_{\mathrm{e}}$ is a reflection around this Hilbert subspace. Operator $U_{\mathrm{o}}$ is defined likewise. The product of those two reflections generates a non trivial unitary operator which defines one step of the coinless quantum walk.

The spatial search is driven by a unitary operator that interlaces the reflection around the marked vertex $U_{w}$ and operators $U_{\mathrm{o}}$ and $U_{\mathrm{e}}$. Patel et al.' choice 9 is $\left(U_{\mathrm{o}} U_{\mathrm{e}}\right)^{3} U_{w}$ while Falk's choice [5] is $U_{\mathrm{o}} U_{w} U_{\mathrm{e}} U_{w}$. Our analytical calculations use the latter one. It is interesting to analyze Patel et al.'s model in order to check their numerical results. Patel et al. briefly discuss the use of the unitary operator $\left(U_{\mathrm{o}} U_{\mathrm{e}}\right)^{t_{1}} U_{w}$ for $t_{1}$ smaller than 3 . It is interesting to analyze the case $t_{1}=1$, which is the simplest one. 
We have analytically shown that the optimal number of steps of the search algorithm is $O(\sqrt{N \log N})$ with a success probability $O(1 / \sqrt{\log N})$ when there is only one marked vertex. We also assumed that $\sqrt{N} / 2$ is odd to simplify the algebraic manipulations. A straightforward application of the method of amplitude amplification provides an algorithm that takes $O(\sqrt{N} \log N)$ steps with success probability $\Theta(1)$. Alternative methods can be explored, such as, classical post-processing search similar to the one proposed by ABNOR 2 . It is also interesting to use of Tulsi's method [12, which is based in the abstract search algorithm described in Ref. [10. Notice that the abstract search algorithm and the coinless search algorithm approximately take place in a two dimensional subspace of the Hilbert space spanned by the initial condition and the marked vertex. It is this fact that is used for obtaining the analytical results of the algorithms.

\section{Acknowledgments}

A.A. and N. N. were supported by EU FP7 project QALGO (FET-Proactive scheme) and

European Research Council grant MQC. R.P. thanks the warm reception at the University of Latvia and acknowledges financial support from CNPq.

\section{References}

[1] Scott Aaronson and Andris Ambainis. Quantum search of spatial regions. In Theory of Computing, pages 200-209, 2003.

[2] A. Ambainis, A. Backurs, N. Nahimovs, R. Ozols, and A. Rivosh. Search by quantum walks on two-dimensional grid without amplitude amplification. 2011, arXiv:1112.3337.

[3] Andris Ambainis, Julia Kempe, and Alexander Rivosh. Coins make quantum walks faster. In Proceedings of the Sixteenth Annual ACM-SIAM Symposium on Discrete Algorithms, SODA, pages 1099-1108, 2005.

[4] Paul Benioff. Space searches with a quantum robot. AMS Contemporary Math Series, 305, 2002.

[5] Matthew Falk. Quantum search on the spatial grid. arXiv:1303.4127, 2013.

[6] Lov K. Grover. Quantum mechanics helps in searching for a needle in a haystack. Phys. Rev. Lett., 79(2):325-328, Jul 1997.

[7] Apoorva Patel, K. S. Raghunathan, and Md. Aminoor Rahaman. Search on a hypercubic lattice using a quantum random walk. ii. $d=2$. Phys. Rev. A, 82:032331, Sep 2010.

[8] Apoorva Patel, K. S. Raghunathan, and Pranaw Rungta. Quantum random walks do not need a coin toss. Phys. Rev. A, 71:032347, Mar 2005.

[9] Apoorva Patel, K. S. Raghunathan, and Pranaw Rungta. Quantum random walks without a coin toss. arXiv:quant-ph/0506221, 2005.

[10] Renato Portugal. Quantum Walks and Search Algorithms. Quantum Science and Technology. Springer, New York, 2013.

[11] N. Shenvi, J. Kempe, and K.B. Whaley. A quantum random walk search algorithm. Phys. Rev. A, 67(5):052307, 2003. quant-ph/0210064.

[12] Avatar Tulsi. Faster quantum-walk algorithm for the two-dimensional spatial search. Physical Review A, 78(1):012310, 2008. 


\section{Appendix}

In this appendix we calculate the eigenvectors and eigenvalues of $U_{1}=U_{\mathrm{e}} U_{w} U_{\mathrm{e}} U_{w}$ and $U_{2}=U_{\mathrm{o}} U_{\mathrm{e}}$, which play an essential role in the determination of the special eigenvector of $U=U_{2} U_{1}$ associated with the eigenvalue with smallest positive argument.

If we suppose that the marked vertex is the origin $|w\rangle=|00\rangle$, the characteristic polynomial of $U_{1}$ is

$$
\left(\lambda^{2}+\lambda+1\right)(\lambda-1)^{N-2} .
$$

In fact, $U_{1}^{3}=I$, which shows that the eigenvalues are $1, \mathrm{e}^{ \pm 2 \pi i / 3}$. The eigenvector associated with $\mathrm{e}^{2 \pi i / 3}$ is

$$
\left|\psi_{1}\right\rangle=\frac{1}{\sqrt{6}}(-i \sqrt{3}|00\rangle+|01\rangle+|10\rangle+|11\rangle),
$$

and $\left|\psi_{2}\right\rangle=\left|\psi_{1}\right\rangle^{*}$ is associated with $\mathrm{e}^{-2 \pi i / 3}$. $U_{1}$ can be expressed as

$$
U_{1}=I+\sqrt{3}\left(\mathrm{e}^{\frac{5 \pi i}{6}}\left|\psi_{1}\right\rangle\left\langle\psi_{1}\left|+\mathrm{e}^{-\frac{5 \pi i}{6}}\right| \psi_{2}\right\rangle\left\langle\psi_{2}\right|\right) .
$$

To obtain the eigenvectors and eigenvalues of $U_{2}$ we use a staggered Fourier transform, which can be introduced in the following form. Define vectors

$$
\left|\Psi_{k l}\right\rangle=a\left|\psi_{k l}^{(0)}\right\rangle+b\left|\psi_{k l}^{(1)}\right\rangle+c\left|\psi_{k l}^{(2)}\right\rangle+d\left|\psi_{k l}^{(3)}\right\rangle,
$$

where

$$
\begin{aligned}
\left|\psi_{k l}^{(0)}\right\rangle & =\frac{2}{\sqrt{N}} \sum_{i, j=0}^{\frac{\sqrt{N}}{2}-1} \omega^{2 i k+2 j l}|2 i, 2 j\rangle, \\
\left|\psi_{k l}^{(1)}\right\rangle & =\frac{2}{\sqrt{N}} \sum_{i, j=0}^{\frac{\sqrt{N}}{2}-1} \omega^{2 i k+(2 j+1) l}|2 i, 2 j+1\rangle, \\
\left|\psi_{k l}^{(2)}\right\rangle & =\frac{2}{\sqrt{N}} \sum_{i, j=0}^{\frac{\sqrt{N}}{2}-1} \omega^{(2 i+1) k+2 j l}|2 i+1,2 j\rangle, \\
\left|\psi_{k l}^{(3)}\right\rangle & =\frac{2}{\sqrt{N}} \sum_{i, j=0}^{\frac{\sqrt{N}}{2}-1} \omega^{(2 i+1) k+(2 j+1) l}|2 i+1,2 j+1\rangle,
\end{aligned}
$$

and $\omega=\mathrm{e}^{2 \pi i / \sqrt{N}}$ and $a, b, c, d$ are complex numbers. For each $k$ and $l,\left|\Psi_{k l}\right\rangle$ span a Hilbert space $\mathcal{H}_{k l}$ that is invariant under the action of $U_{2}$. $U_{2}$ can be expressed as a reduced $4 \times 4$-matrix,

$$
U_{2}^{\mathrm{red}}=\left[\begin{array}{cccc}
\frac{\cos \tilde{k} \cos \tilde{l}}{\omega^{k+l}} & \frac{\sin \tilde{k} \cos \tilde{l}}{i \omega^{k}} & \frac{\cos \tilde{k} \sin \tilde{l}}{i \omega^{l}} & \sin \tilde{k} \sin \tilde{l} \\
\frac{\sin \tilde{k} \cos \tilde{l}}{i \omega^{k}} & \frac{\omega^{l} \cos \tilde{k} \cos \tilde{l}}{\omega^{k}} & -\sin \tilde{k} \sin \tilde{l} & i \omega^{l} \cos \tilde{k} \sin \tilde{l} \\
\frac{\cos \tilde{k} \sin \tilde{l}}{i \omega^{l}} & -\sin \tilde{k} \sin \tilde{l} & \frac{\omega^{k} \cos \tilde{k} \cos \tilde{l}}{\omega^{l}} & i \omega^{k} \sin \tilde{k} \cos \tilde{l} \\
\sin \tilde{k} \sin \tilde{l} & i \omega^{l} \cos \tilde{k} \sin \tilde{l} & i \omega^{k} \sin \tilde{k} \cos \tilde{l} & \omega^{k+l} \cos \tilde{k} \cos \tilde{l}
\end{array}\right],
$$

where $\tilde{k}=\frac{2 \pi k}{\sqrt{N}}$ and $\tilde{l}=\frac{2 \pi l}{\sqrt{N}}$. $U_{2}^{\text {red }}$ can be diagonalized and the eigenvalues and eigenvectors of this reduced matrix can be used to obtain the eigenvalues and eigenvectors of $U_{2}$ in the original Hilbert space. The eigenvalues of $U_{2}^{\text {red }}$ are 1 and $\mathrm{e}^{ \pm i \theta}$, where

$$
\cos \theta=2 \cos ^{2} \tilde{k} \cos ^{2} \tilde{l}-1 .
$$


Note that $\theta$ depends on $k, l$, and $N$. The normalized eigenvectors associated with eigenvalue 1 are

$$
\left|w_{k l}^{(0)}\right\rangle=\frac{1}{2 c^{+}}\left[\begin{array}{c}
\sin (\tilde{k}-\tilde{l}) \\
\sin \tilde{l}-\sin \tilde{k} \\
\sin \tilde{l}-\sin \tilde{k} \\
\sin (\tilde{k}-\tilde{l})
\end{array}\right], \quad\left|w_{k l}^{(1)}\right\rangle=\frac{1}{2 c^{-}}\left[\begin{array}{c}
\sin (\tilde{l}-\tilde{k}) \\
\sin \tilde{k}+\sin \tilde{l} \\
-\sin \tilde{k}-\sin \tilde{l} \\
\sin (\tilde{k}-\tilde{l})
\end{array}\right],
$$

where $\left(c^{ \pm}\right)^{2}=(1 \pm \cos \tilde{k} \cos \tilde{l})(1 \mp \cos (\tilde{k}-\tilde{l}))$. When $k=l$, the first eigenvector reduces to

$$
\left|w_{k k}^{(0)}\right\rangle=\frac{1}{\sqrt{2} \sqrt{1+\cos ^{2} \tilde{k}}}\left[\begin{array}{c}
1 \\
-\cos \tilde{k} \\
-\cos \tilde{k} \\
1
\end{array}\right]
$$

and the second eigenvector reduces to $\left|w_{k k}^{(1)}\right\rangle=[0,1 / \sqrt{2},-1 / \sqrt{2}, 0]$. Using the fact that $\left\langle\psi_{0} \mid \psi_{k l}^{ \pm \pm}\right\rangle=\frac{1}{2} \delta_{k, 0} \delta_{l, 0}$, it is straightforward to show that the inner products between the initial condition and all those eigenvectors are zero. Using the fact that $\left\langle 00 \mid \psi_{k l}^{(0)}\right\rangle=\frac{2}{\sqrt{N}}$ and $\left\langle 00 \mid \psi_{k l}^{(1)}\right\rangle=\left\langle 00 \mid \psi_{k l}^{(2)}\right\rangle=\left\langle 00 \mid \psi_{k l}^{(3)}\right\rangle=0$, it is straightforward to calculate the inner products between the target state $|00\rangle$ and those eigenvectors, which are

$$
\begin{aligned}
\left\langle 00 \mid w_{k l}^{(0)}\right\rangle & =\frac{\sin (\tilde{k}-\tilde{l})}{c^{+} \sqrt{N}} \\
\left\langle 00 \mid w_{k l}^{(1)}\right\rangle & =\frac{\sin (\tilde{l}-\tilde{k})}{c^{-} \sqrt{N}}
\end{aligned}
$$

When $k=l$ the inner product between the target and those eigenvectors are $\left\langle 00 \mid w_{k k}^{(0)}\right\rangle=$ $\sqrt{2} /\left(\sqrt{N} \sqrt{1+\cos ^{2} \tilde{k}}\right)$ and $\left\langle 00 \mid w_{k k}^{(1)}\right\rangle=0$.

The normalized eigenvectors associated with eigenvalue $\mathrm{e}^{i \theta}$ are

$$
\left|w_{k l}^{(2)}\right\rangle=\frac{1}{2 c}\left[\begin{array}{c}
-\epsilon \sqrt{c-\epsilon \sin \tilde{k} \cos \tilde{l}} \sqrt{c-\epsilon \cos \tilde{k} \sin \tilde{l}} \\
\sqrt{c-\epsilon \sin \tilde{k} \cos \tilde{l}} \sqrt{c+\epsilon \cos \tilde{k} \sin \tilde{l}} \\
\sqrt{c+\epsilon \sin \tilde{k} \cos \tilde{l}} \sqrt{c-\epsilon \cos \tilde{k} \sin \tilde{l}} \\
\epsilon \sqrt{c+\epsilon \sin \tilde{k} \cos \tilde{l}} \sqrt{c+\epsilon \cos \tilde{k} \sin \tilde{l}}
\end{array}\right],
$$

where $c^{2}=1-\cos ^{2} \tilde{k} \cos ^{2} \tilde{l}$ and $\epsilon$ is the $\operatorname{sign}$ of $\cos \tilde{k} \cos \tilde{l}$. Note that $c \geq \sin \tilde{k} \cos \tilde{l}$. When $k=l$, they reduce to

$$
\left|w_{k k}^{(2)}\right\rangle=\frac{1}{2 \sqrt{1+\cos ^{2} \tilde{k}}}\left[\begin{array}{c}
\cos \tilde{k}-\epsilon \sqrt{1+\cos ^{2} \tilde{k}} \\
1 \\
1 \\
\cos \tilde{k}+\epsilon \sqrt{1+\cos ^{2} \tilde{k}}
\end{array}\right] .
$$

Using the fact that the entries of $\left|w_{k l}^{(2)}\right\rangle$ are real and $\left(U_{2}^{\mathrm{red}}\right)^{*}=M U_{2}^{\mathrm{red}} M$, where

$$
M=\left[\begin{array}{llll}
0 & 0 & 0 & 1 \\
0 & 0 & 1 & 0 \\
0 & 1 & 0 & 0 \\
1 & 0 & 0 & 0
\end{array}\right]
$$


we show that the eigenvectors associated with eigenvalue $\mathrm{e}^{-i \theta}$ are obtained by inverting the lines of the eigenvectors associated with $\mathrm{e}^{i \theta}$. Instead of inverting the entries of eigenvector, one can invert the sign of $\epsilon$. Notice that for some values of $k$ and $l$ eigenvalues $\mathrm{e}^{ \pm i \theta}$ can be 1. Also notice that eigenvalues $\mathrm{e}^{ \pm i \theta}$ can be -1 only if $\sqrt{N} / 2$ is even.

The eigenvectors of the full matrix are

$$
\left|v_{k l}^{(\beta)}\right\rangle=\sum_{\beta^{\prime}=0}^{3}\left\langle\beta^{\prime} \mid w_{k l}^{(\beta)}\right\rangle\left|\psi_{k l}^{\left(\beta^{\prime}\right)}\right\rangle,
$$

for $\beta=0, \ldots, 3$ and $0 \leq k, l<\sqrt{N} / 2$, and the eigenvalues are $w_{k l}^{(0)}=w_{k l}^{(1)}=1, w_{k l}^{(2)}=\mathrm{e}^{i \theta}$, and $w_{k l}^{(3)}=\mathrm{e}^{-i \theta}$. The entries of $\left|w_{k l}^{(\beta)}\right\rangle$ are represented by $\left\langle\gamma \mid w_{k l}^{(\beta)}\right\rangle, 0 \leq \gamma \leq 3$, to avoid confusion with the notation of the eigenvalues. Notice that $\left|v_{k l}^{(2)}\right\rangle$ and $\left|v_{k l}^{(3)}\right\rangle$ are not complex conjugate. In order to check the results that depend on Eqs. (13) and (14), we have to replace $\left|v_{k l}^{(3)}\right\rangle$ by the complex conjugate of $\left|v_{k l}^{(2)}\right\rangle$.

We can decompose $\left|\psi_{1}\right\rangle$ in the eigenbasis of $U_{2}$ as

$$
\left|\psi_{1}\right\rangle=\sum_{k, l=0}^{\frac{\sqrt{N}}{2}-1} \sum_{\beta=0}^{3} a_{k l}^{(\beta)}\left|v_{k l}^{(\beta)}\right\rangle,
$$

where

$$
a_{k l}^{(\beta)}=\frac{2}{\sqrt{N}}\left\langle w_{k l}^{(\beta)} \mid \psi_{1}^{\mathrm{red}}\right\rangle
$$

and

$$
\left|\psi_{1}^{\text {red }}\right\rangle=\frac{1}{\sqrt{6}}\left[\begin{array}{c}
-i \sqrt{3} \\
\omega^{-l} \\
\omega^{-k} \\
\omega^{-(k+l)}
\end{array}\right]
$$

The details of the calculation of $|a|^{2}$, which can be obtained from Eq. (13), are

$$
\begin{aligned}
|a|^{2} & =\sum_{k, l=0}^{\frac{\sqrt{N}}{2}-1}\left(\left|a_{k l}^{(0)}\right|^{2}+\left|a_{k l}^{(1)}\right|^{2}\right)+\left|a_{00}^{(2)}\right|^{2}+\left|a_{00}^{(3)}\right|^{2} \\
& =\frac{1}{3}+\frac{10}{3 N}-\frac{4}{3 N} \sum_{k, l=0}^{\frac{\sqrt{N}}{2}-1} \frac{\cos \tilde{k} \sin \tilde{k} \cos \tilde{l} \sin \tilde{l}}{1-\cos ^{2} \tilde{k} \cos ^{2} \tilde{l}} \\
& =\frac{1}{3}+\frac{8}{3 N}+O\left(\frac{1}{N^{2}}\right) .
\end{aligned}
$$

